\title{
Borehole Electromagnetic Method for Exploration of Coal Mining Goaf
}

\author{
Benyu $\mathrm{Su}^{1,2}$, Jingcun $\mathrm{Yu}^{1,2}$, Chenxing Sheng ${ }^{3}$ \\ ${ }^{1}$ Institute of Applied Geophysics, School of Resource \& Earth Science, China University of Mining and \\ Technology, \\ University St. 1, 221116 Xuzhou, China \\ ${ }^{2}$ State Key Laboratory for Geomechanics \& Deep Underground Engineering, China University of \\ Mining and Technology, \\ University St. 1, 221116 Xuzhou, China \\ ${ }^{3}$ School of Power \& Energy Engineering, Wuhan University of Technology, \\ Peace St. 170, 430063 Wuhan, China \\ schxing@yeah.net
}

\begin{abstract}
Due to severe harms of goaf collapse, the goaf exploration and governance has become an urgent issue for protecting the normal life of local people. According to the coal mine geology, different geo-electrical models have been employed for the purpose of discovering the goafs. However, most existing methods require a large amount of computation consumption. In order to address this issue, a forward numerical simulation using the borehole electromagnetic method has been developed in this work to explore the coal mining goafs. The innovation of this method is that the computation consuming can be saved significantly. Numerical simulation demonstrates high effectiveness of the borehole electromagnetic method in coal mining goaf exploration. Therefore, this research provides a new idea for exploring the coal mine goafs by geophysical method.
\end{abstract}

Index Terms-Borehole electromagnetic; geophysical measurement techniques; geophysics computing; environment geophysics; coal mining goaf.

\section{INTRODUCTION}

In the past few years, a lot of geological disasters which have been caused by collapse of mining goafs, as shown in Fig. 1. These collapses of mining goafs led to a great economic loss [1] and casualties as well as environmental deterioration [2]. Furthermore, these collapses of mining goafs brought a lot of panics to the local people [3]. Hence, investigation of depth and range of mining goafs has a great meaningful significance for treatment of mining goafs so as to recover the normal life for local people.

At present, many geophysical methods have been adopted to prospect coal mining goaf. However, each method has its own disadvantage which limits the validity for prospecting coal mining goafs, particularly for multilayer goafs. In this paper, a borehole electromagnetic method is developed to explore the coal mining goafs.

Since the 1970s, the forward modelling of geophysical

Manuscript received 3 December, 2015; accepted 9 April, 2016.

This research was funded by the Fundamental Research Funds for the Central Universities (NO. 2014QNA88) from China University of Mining and Technology. electromagnetic method has been proposed and the results have been published. Hohmann [4], Wannamaker [5] and Wei et al. [6] calculated three dimensional models by integral equation method. Pridmore et al. [7] simulated the responses of abnormities by finite element method in the horizontal layered medium. Because integral equation method only needs to discretize the bodied of abnormities, it has better preciseness, less unknown variables and it is convenient to be used in practice. However, integral equation method needs to solve the reverse of large complex matrix directly; therefore it needs high computing speed and large internal memory of computer.

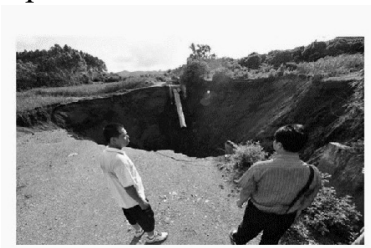

a)

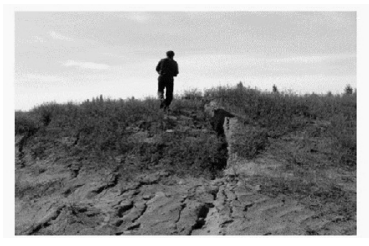

c)

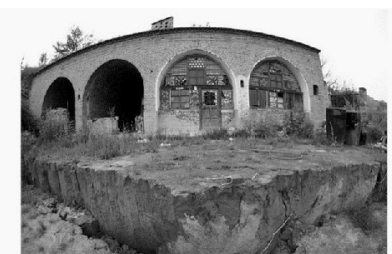

b)

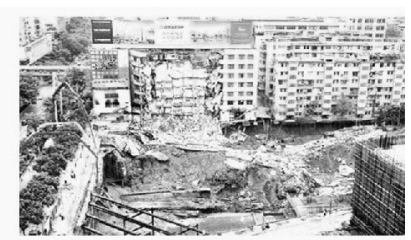

d)
Fig. 1. Coal mining goafs: (a) the harm caused by the goaf, (b) the harm to rural residence, (c) the harm on natural environment and (d) the harm to urban buildings.

Finite difference method and finite element method are based on partial differential equation. Hence, theoretically these two methods can solve any complex model. However, it has to discretize the entire model; as a result, it needs high performance of computers [8], [9]. Alumbaugh et al. [10] obtained a good result of electromagnetic responses using staggered-grid finite difference method by parallel computing. Because of limitation of computer resource, staggered-grid finite difference method which was developed by Yee [11] has not been employed in the geophysical field until 1980s. 
Recently, Alvarez-Aramberri et al. [12] proposed an hp-finite element method for multi-goal magnetotelluric measurements to save computational cost. Wang et al. [13] conducted experimental measurements in different boreholes to demonstrate the application of the electrokinetic logging for the oil exploration. Yang et al. [14] used the finite-difference time-domain (FDTD) method to simulate the outstanding problem in acoustic logging while drilling (LWD). Tietze et al. [15] applied the controlled source electromagnetic (CSEM) to monitor fluid movements. Streich [16] reviewed previous and present approaches about the controlled-source electromagnetics (CSEM) for hydrocarbon exploration and monitoring. Zhao et al. [17] verified the coal-fire areas using the electrical resistance tomography. However, to date, quite limited work has addressed the issue that using the borehole electromagnetic method to solve the problem of exploring the goafs. In order to solve this problem, a practical approach using staggered grid finite difference method is presented in this paper. The contribution of this work is that a borehole electromagnetic method has been developed to prospect coal mining goafs. Numerical simulations have been carried out to verify the effectiveness of the proposed method.

\section{Methodology}

In this paper, electromagnetic responses of medium are obtained by solving Maxwell equation using staggered grid finite difference method. In the process of discretization, three dimensional medium is discretized into the unit cube, and every unit cube is a uniform electrical body. In addition, electric field components are set on the middle of the cubic edge, while magnetic field components are set in the centre of the side face, as shown in Fig. 2.

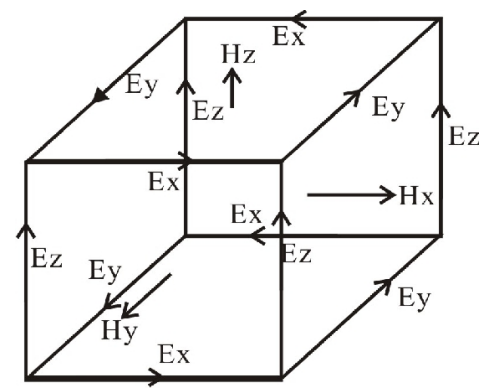

Fig. 2. Staggered grid and the distribution of the field.

It is assumed that the change of electromagnetic field with the terms of $\exp (i \omega \mathrm{t})$, here, $i=\sqrt{-1}$ and $\omega$ is circular frequency: $\omega=2 \pi f$. In the domain of frequency, the Maxwell equation [13] can be expressed:

$$
\begin{gathered}
\nabla \times E=-i \omega \mu H-M_{p}, \\
\nabla \times H=i \omega\left[\varepsilon-\mathrm{i} \frac{\sigma}{\omega}\right] E+J_{p},
\end{gathered}
$$

where $\mu$ is the magnetic permeability: $\mu=4 \pi \times 10^{-7} \mathrm{H} / \mathrm{m}$; $\varepsilon$ is the dielectric constant: $\varepsilon=\varepsilon_{r} \varepsilon_{0}$, here $\varepsilon_{r}$ is the relative dielectric constant, and $\varepsilon_{0}$ is the air dielectric constant; $\sigma$ is the electric conductivity; $E$ is the electric field and $H$ is the magnetic field; $J_{p}$ is the density of electric current and $M_{p}$ is the density of magnetic current. In the following discussion, taking magnetic dipole $\left(J_{p} \neq 0.0\right.$ and $M_{p}=0.0$ ) has been taken as an example to deduce discretized Maxwell equation.

Based on (1) and (2), the Helmholtz equation can be obtained

$$
\nabla \times \nabla \times E-k_{t}^{2} E=J_{p}
$$

In order to simplify computation and improve calculation accuracy, the total electric field $(E)$ is divided into background field $\left(E_{b}\right)$ and the second field $\left(E_{s}\right)$

$$
E=E_{b}+E_{s}
$$

Equation (5) is obtained by (3) and (4)

$$
\nabla \times \nabla \times E_{s}-k_{t}^{2} E_{s}=\left(k_{t}^{2}-k_{b}^{2}\right) E_{b},
$$

where the background electric field $E_{b}$ is the analytical solution which can be solved by the analytical (7) [14]; the second electric field $\left(E_{s}\right)$ is calculated by staggered-grid finite difference method; $k_{b}$ is the complex wave number of background medium and it can be expressed by (6):

$$
\begin{gathered}
k_{b}^{2}=\omega^{2} \mu\left[\varepsilon_{p}-i \frac{\sigma_{b}}{\omega}\right], \\
E_{b}=i \omega \mu M \frac{r e^{-i k_{b} R}}{4.0 \pi(R)^{3}}\left(1+i k_{b} R\right) .
\end{gathered}
$$

In the (7), $r$ represents the inter-well distance; $z$ represents vertical offset; $R$ can be calculated by $R=\sqrt{r^{2}+z^{2}} ; M$ is the magnetic dipole moment; $\omega$ is the angle frequency and $\mu$ is the permittivity. For the calculation of the secondary field $\left(E_{s}\right)$, (5) is divided into three components. Three electric fields in direction of $x, y$ and $z$ are represented by:

$$
\begin{gathered}
\frac{\partial^{2} E_{y}}{\partial x \partial y}+\frac{\partial^{2} E_{z}}{\partial z \partial x}-\frac{\partial^{2} E_{x}}{\partial y^{2}}-\frac{\partial^{2} E_{x}}{\partial z^{2}}-k_{t x}^{2} E_{x}= \\
=\left(k_{t x}^{2}-k_{b}^{2}\right) E_{x p}, \\
\frac{\partial^{2} E_{z}}{\partial y \partial z}+\frac{\partial^{2} E_{x}}{\partial x \partial y}-\frac{\partial^{2} E_{y}}{\partial z^{2}}-\frac{\partial^{2} E_{y}}{\partial x^{2}}-k_{t y}^{2} E_{y}= \\
=\left(k_{t y}^{2}-k_{b}^{2}\right) E_{y p}, \\
\frac{\partial^{2} E_{x}}{\partial z \partial x}+\frac{\partial^{2} E_{y}}{\partial y \partial z}-\frac{\partial^{2} E_{z}}{\partial x^{2}}-\frac{\partial^{2} E_{z}}{\partial y^{2}}-k_{t z}^{2} E_{z} \\
=\left(k_{t z}^{2}-k_{b}^{2}\right) E_{z p},
\end{gathered}
$$

where $k_{t x}, k_{t y}$ and $k_{t z}$ are the complex wave numbers in the directions of $x, y$ and $z$, respectively. According to the above discussion, the matrix equation can be obtained by

$$
A X=B
$$


where $A$ is a sparse matrix with the following structure

$$
A=\left|\begin{array}{ccc}
A_{x x} & A_{x y} & A_{x z} \\
A_{y x} & A_{y y} & A_{y z} \\
A_{z x} & A_{z y} & A_{z z}
\end{array}\right|,
$$

where $A_{x x}, A_{x y}, A_{x y}, A_{y x}, A_{y y}, A_{y z}$ and $A_{z x}, A_{z y}, A_{z z}$ are coefficient matrixes by discrete (8)-(10), respectively; $B$ is the equivalent source matrix which is the product by complex wave number and background field, as shown in the right parts of (8)-(10); $X$ is the electric field matrix

$$
\begin{aligned}
X= & {\left[E_{x}(1), E_{x}(2), \cdots, E_{x}\left(N_{x}\right), E_{y}(1), E_{y}(2),\right.} \\
& \left.\cdots, E_{y}\left(N_{y}\right), E_{z}(1), E_{z}(2) \cdots, E_{z}\left(N_{z}\right)\right] .
\end{aligned}
$$

Due to $A$ being as a sparse matrix, the bandwidth of non-zero matrix storage technique is adopted to store the non-zero element of the matrix $A$. In this storage technique, a one-dimensional array $(A D(I))$ and a bandwidth of two-dimensional array $(A U(J, I))$ are employed to storage diagonal elements and the elements in the right-upper triangle respectively. In addition, we use anther two-dimensional array $(I U(J, I))$ to record the address of non-zero elements in right-upper triangle and the numbers of non-zero elements in the Ith row is recorded in the one-dimensional array $(N Z U(I))$. The bandwidth of non-zero matrix storage technique can be described in details below:

1. The number of non-zero elements in the Ith row on the right of diagonal is recorded in the array of $N Z U(I)$;

2 . The column number of the $J$ th non-zero element on the right of diagonal in the $I$ th row is recorded in the array of $I U(J, I)$;

3. The element corresponding to the column number recorded in the array of $I U(J, I)$ in the $I$ th row is stored in the array of $A U(J, I)$;

4. The diagonal elements are stored in the array of $A D(I)$.

In the sparse matrix, up to $95 \%$ of storage space is occupied by zero elements, hence the storage technique of sparse matrix is critical for fast solving equations and dealing with practical problem.

For solving equation $A$ to calculate electric field $E$, we adopt Incompletely Cholesky Conjugate Gradient method
(Kershaw, 1978) to solve matrix equations. Finally, the magnetic fields are calculated by (14)-(16):

$$
\begin{aligned}
& H_{x}=\frac{i}{\omega \mu}\left(\frac{\partial E_{y}}{\partial z}-\frac{\partial E_{z}}{\partial y}\right), \\
& H_{y}=\frac{i}{\omega \mu}\left(\frac{\partial E_{z}}{\partial x}-\frac{\partial E_{x}}{\partial z}\right), \\
& H_{z}=\frac{i}{\omega \mu}\left(\frac{\partial E_{x}}{\partial y}-\frac{\partial E_{y}}{\partial x}\right) .
\end{aligned}
$$

\section{NUMERICAL SIMULATION}

Model 1 was designed to examine the feasibility and effectiveness of the methodology, as shown in Fig. 3. With purpose of simulating the borehole electromagnetic method, the vertical magnetic dipoles were set in the arbitrary point in the model. In Fig. 3(a), there were two goafs for numerical simulation and the upper one indicated a low resistivity goaf filled with water while the other one is a high resistivity goaf filled with air. Generally, for the resistivity of the goaf filling with water, its resistivity value is usually about $1 \mathrm{ohm}-\mathrm{m}$. However, for the goaf filling with air, its resistivity value is about $1000 \mathrm{ohm}-\mathrm{m}$. Hence, in Fig. 3(a) the resistivities of two goafs were $1 \mathrm{ohm}-\mathrm{m}$ and $1000 \mathrm{ohm}-\mathrm{m}$, respectively. The background resistivity was $100 \mathrm{ohm}-\mathrm{m}$. In the geo-electrical model, the small star schema indicated the source point. According to the model, we calculated the magnetic field for every node. In order to highlight the abnormal response, magnetic fields are transformed into the amplitude difference between total magnetic field and background magnetic field. And Fig. 3(b) is the slice which is cut from 3D data volume. Comparing Fig. 3(a) with Fig. 3(b), the forward simulation results correctly reflect the positions of the two goafs.

Model 2 was adopted to simulate the cross-hole prospecting, as shown in Fig. 4. And the resistivities of the two goafs were $5 \mathrm{ohm}-\mathrm{m}$ and $10 \mathrm{ohm}-\mathrm{m}$, respectively. The resistivity of background was $100 \mathrm{ohm}-\mathrm{m}$. In order to make the effectiveness to be obvious, the modeling results were standardized using (17)

$$
H z z=\frac{H z}{H b},
$$

where $H z$ denotes the total magnetic field, $H b$ is the background field, and $\mathrm{Hzz}$ is the standardized result.

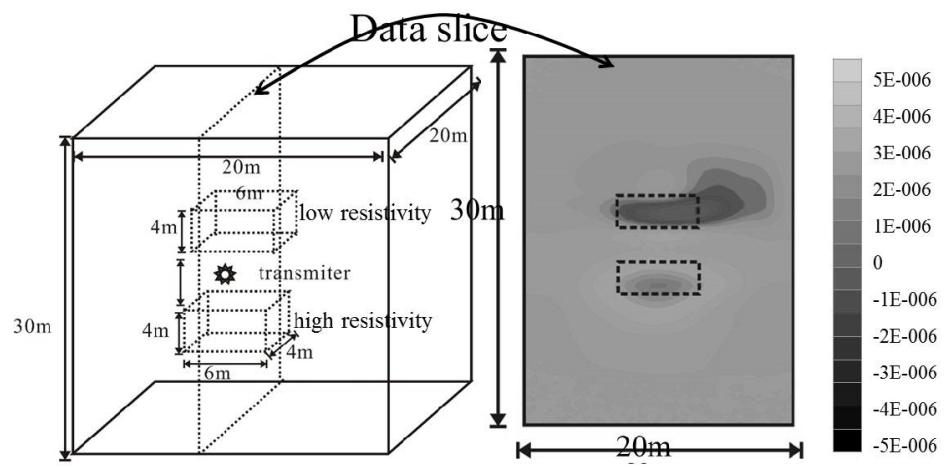

a)

b)

Fig. 3. The model of numerical simulation (a) and the detection results (b). There are two goafs in the model and the upper one is supposed as the goaf filled with water; however, the lower one is to be empty. 


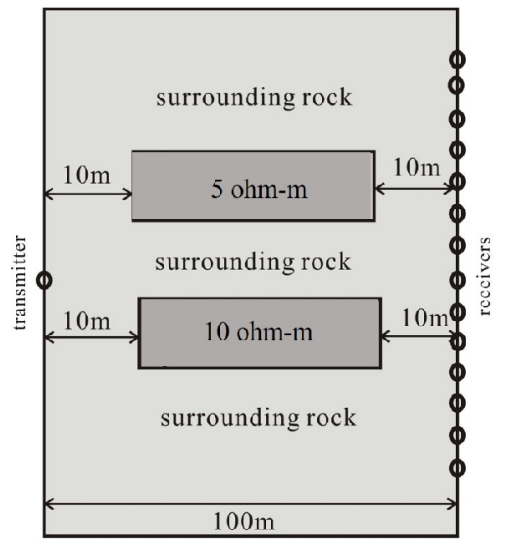

a)

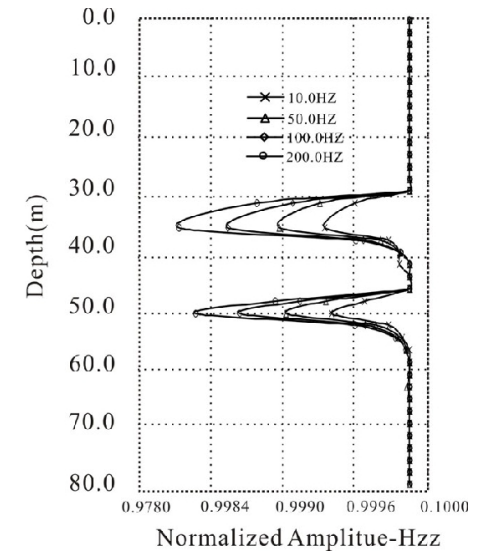

b)

Fig. 4. The cross-hole model (a) and the detecting result (b). There are two goafs in the model and the upper one is supposed the resistivity of 5 ohm-m and the lower one is $10 \mathrm{ohm}-\mathrm{m}$. In addition, the background resistivity is $100 \mathrm{ohm}-\mathrm{m}$.

In this study, the modeling result was exhibited by standardized amplitude, as shown in Fig. 4(b). In additional, different sources with different frequencies were adopted to investigate the resolution for different frequencies. In the numerical simulation, sources with four different frequencies were adopted and the modeling results are shown in Fig. 4 (b). Compared with four different modeling results, the resolutions will enhance with the frequency increase.

\section{CONCLUSIONS}

Since it is difficult to explore multilayer goafs by common geophysical method, a borehole electromagnetic method is developed in this work to address this issue. The finite different method has been employed by the borehole electromagnetic method. With the purpose of examining the performance of the present method for prospecting multilayer goaf, two geo-electrical models are adopted to in the numerical simulations. The numerical analysis results show that the borehole electromagnetic method is valid for prospecting multilayer goaf. In addition, the relationship between resolution and source frequencies is studied using four different frequencies source and the resolution will become high with increasing of frequency.

\section{REFERENCES}

[1] Y. Jiang, H. Zhu, Z. Li, “A new compound faults detection method for rolling bearings based on empirical wavelet transform and chaotic oscillator", Chaos, Solitons and Fractals, 2015. [Online]. Available: http://dx.doi.org/10.1016/j.chaos.2015.09.007

[2] Y. Jiang, H. Zhu, Z. Li, Z. Peng, "The nonlinear dynamics response of cracked gear system in a coal cutter taking environmental multi-frequency excitation forces", Nonlinear Dynamics, vol. 84, pp. 203-222, 2016. [Online]. Available: http://dx.doi.org/10.1007/ s11071-015-2409-2

[3] Z. Li, Y. Jiang, X. Wang, Z. Peng, "Multi-mode separation and nonlinear feature extraction of hybrid gear failures in coal cutters using adaptive nonstationary vibration analysis", Nonlinear Dynamics, vol. 84, pp. 295-310, 2016. [Online]. Available: http://dx.doi.org/ 10.1007/s11071-015-2505-3

[4] G. W. Hohmann, "Three-dimensional induced polarization and electromagnetic modeling", Geophysics, vol. 40, pp. 309-324, 1975. [Online]. Available: http://dx.doi.org/10.1190/1.1440527

[5] P. E. Wannamaker, G. W. Hohmann, W. A San Fulipo, "Electromagnetic modeling of three-dimensional bodies in layered earths using integral equations", Geophysics, vol. 49, pp. 60-74, 1984. [Online]. Available: http://dx.doi.org/10.1190/1.1441562

[6] B. J. Wei, G. Y. Zhang, "Forward modeling and inversion of 3-D cross-hole electromagnetic fields", Chinese Journal of Geophysics, vol. 45, pp. 735-743, 2002. [Online]. Available: http://dx.doi.org/ $10.1002 /$ cjg2.291

[7] D. F. Pridmore, G. W. Hohmann, S. H. Ward, et al., "An investigation of finite element modeling for electrical and electromagnetic data in three dimensions", Geophysics, vol. 46, pp. 1009-1024, 1981. [Online]. Available: http://dx.doi.org/10.1190/1.1441239

[8] D. L. Alumbaugh, H. F. Morrison, "Monitoring subsurface changes over time with cross-well electromagnetic tomography", Geophysical Prospecting, vol. 43, pp. 873-902, 1995. [Online]. Available: http://dx.doi.org/10.1111/j.1365-2478.1995.tb00286.x

[9] K. S. Yee, "Numerical solution of initial boundary value problems involving Maxwell's equations in isotropic media", IEEE Trans. Antennas Propagation, vol. 14, pp. 302-309, 1966. [Online]. Available: http://dx.doi.org/10.1109/TAP.1966.1138693

[10] J. S. Shen, "Modeling of 3-D electromagnetic responses in frequency domain by using staggered-grid finite difference method", Chinese Journal of Geophysics, vol. 46, pp. 281-289, 2003. [Online]. Available: http://dx.doi.org/10.1002/cjg2.355

[11] W. C. Chew, W. H. Weedom, "A 3-D perfectly matched medium from modified Maxwell's equations with stretched coordinates", Microwave and Optical Technology Letters, vol. 7, pp. 599-604, 1994. [Online]. Available: http://dx.doi.org/10.1002/mop.465007 1304

[12] J. Alvarez-Aramberri, D. Pardo, H. Barucq, "A secondary field based hp-Finite Element Method for the simulation of magnetotelluric measurements", Journal of Computational Science, vol. 11, pp. 137-144, 2015. [Online]. Available: http://dx.doi.org/10.1016/ j.jocs.2015.02.005

[13] J. Wang, W. Guan, H. Hu, Z. Zhu, "Electrokinetic experimental studies in borehole model II: localized and radiated seismoelectric field", Chinese Journal of Geophysics, vol. 59, pp. 381-390, 2016.

[14] Y. Yang, W. Guan, N. Cui, H. Hu, X. Zheng, "FDTD simulation and analysis of the collar wave propagation in acoustic logging while drilling", Chinese Journal of Geophysics, vol. 59, pp. 368-380, 2016.

[15] K. Tietze, O. Ritter, P. Veeken, "Controlled-source electromagnetic monitoring of reservoir oil saturation using a novel borehole-to-surface configuration", Geophysical Prospecting, vol. 63, pp. 1468-1490, 2015. [Online]. Available: http://dx.doi.org/10.1111/13652478.12322

[16] R. Streich, "Controlled-source electromagnetic approaches for hydrocarbon exploration and monitoring on land", Surveysin Geophysics, vol. 37, pp. 47-80, 2016. [Online]. Available: http://dx.doi.org/10.1007/s10712-015-9336-0

[17] Z. Shao, D. Wang, Y. Wang, X. Zhong, X. Tang, D. Xi, "Electrical resistivity of coal-bearing rocks under high temperature and the detection of coal fires using electrical resistance tomography", Geophysical Journal International, vol. 204, pp. 1316-1331, 2016. [Online]. Available: http://dx.doi.org/10.1093/gji/ggv525 